EDUCATION

\title{
From catastrophe to complexity: a novel model of integrative central neural regulation of effort and fatigue during exercise in humans
}

\author{
T D Noakes, A St Clair Gibson, E V Lambert
}

Br J Sports Med 2004;38:51 1-514. doi: 10.1136/bjsm.2003.009860

It is a popular belief that exercise performance is limited by metabolic changes in the exercising muscles, so called peripheral fatigue. Exercise terminates when there is a catastrophic failure of homoeostasis in the exercising muscles. A revolutionary theory is presented that proposes that exercise performance is regulated by the central nervous system specifically to ensure that catastrophic physiological failure does not occur during normal exercise in humans.

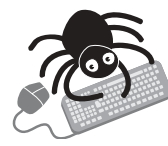

This paper forms part of a symposium series. Each of the four accompanying papers will be published in the journal over the next few issues, and the full series will be accessible at http://bjsm.bmijournals. com/supplemental

See end of article for authors' affiliations

Correspondence to: Professor Noakes, University of Cape Town, Research Unit for Exercise Science and Sports Medicine, Sports Science Institute of South Africa, PO Box 115, Newlands 7725, South Africa; tdnoakes@sports.uct.ac.za

Accepted 4 May 2004
C ertain truths are self evident; others are more deeply hidden. Perhaps the most interesting question in exercise physiology is the one that is not often asked: why do muscles not develop rigor during exercise of high intensity and short duration (seconds to minutes) or when their fuel reserves are depleted during more prolonged exercise lasting two or more hours? Consider for example, the following. Human skeletal muscle can increase its peak rate of ATP utilisation to about $3 \mu \mathrm{mol} / \mathrm{s} / \mathrm{kg}$ wet weight within seconds of beginning intensive exercise. ${ }^{1}$ Yet skeletal muscle ATP concentrations are $5-7 \mu \mathrm{mol} / \mathrm{kg}$ wet weight. ${ }^{2}$ Thus as little as seven seconds of peak exercise would deplete the muscle ATP stores and induce rigor as a catastrophic event if the peak rate of muscle ATP production was less than $80 \%$ of demand. Indeed, Newsholme and Start ${ }^{2}$ have calculated that total ATP stores in the flight muscles of the fly, Lucilia sericata, would sustain about 0.1 second of flight, and the white leg muscles of the rabbit about 1.0 second of running respectively if other mechanisms for instantaneous rephosphorylation of ADP did not exist. exercise in any vertebrate, the rates of ATP use and production must be perfectly matched, even during maximum exercise in all members of the animal kingdom. As a result, ATP concentrations in maximally exercising muscles fall relatively little during exercise, ${ }^{3}$ even in muscles that are deficient in certain key metabolic enzymes which would impair their capacity to generate ATP, especially the group of conditions collectively termed McArdle's syndrome. ${ }^{4}$ Thus it appears that muscle ATP concentrations during exercise are homoeostatically regulated with exquisite accuracy, as they are at rest. This has to be so because, under physiological conditions, the flow
As muscle rigor has yet to be described during of electrons from carbohydrate and fats is tightly coupled to the synthesis of ATP in the mitochondria. During muscle contraction, the resulting increase in ADP concentrations causes an increase in the rate of oxidative phosphorylation, resulting in the resynthesis of ATP, and preventing a dramatic decrease in ATP concentrations in the muscle.

Yet some of the traditional explanations of how fatigue develops during both maximal and more prolonged exercise in humans seemingly disregard this reality. For the popular theories posit that fatigue develops, and exercise terminates, whenever the rate of ATP use by the exercising muscles exceeds the maximum available rate of oxidative ATP production of those muscles. Thus it is believed that, during high intensity exercise, the shortfall in oxidative ATP production is supplemented by the less sustainable "anaerobic" ATP production, leading to fatigue when the rate of ATP production becomes less than its rate of ATP use. During more prolonged exercise, it is proposed that the shortfall in oxidative ATP production cannot be remedied. ${ }^{6}$ Rather the muscles "fatigue" so that either the exercise must be terminated or it can continue only at lower intensities.

These concepts are based on the "limitations" or "catastrophe" models of exercise physiology, which conjecture that fatigue develops only after one or more bodily systems are stressed beyond their capacity, leading rapidly to the complete system failure that we recognise as exhaustion. The most long standing example, perhaps the earliest source of this intellectual mindset, is the theory that a limiting oxygen delivery to muscle, leading to skeletal muscle hypoxia or anaerobiosis, is the cause of fatigue during high intensity exercise of short duration, the so called cardiovascular/anaerobic/catastrophic model of exercise physiology. ${ }^{8}$

However, the overlooked flaw in this explanation is that fatigue cannot be the immediate consequence of an imbalance in the rates of muscle ATP production and ATP use. For, if that were true, as soon as the rate of ATP production lags behind its rate of use, muscle ATP concentrations must fall inexorably, leading ultimately to muscle rigor. ${ }^{3}$ But, if muscle rigor does not develop during any form of exercise in vertebrates, then some other control mechanism must exist to terminate exercise even though the rates of skeletal muscle ATP production and use are in balance and skeletal muscle ATP concentrations are sufficiently high to prevent the development of rigor. 
In a symposium at the 2001 American College of Sports Medicine Annual Conference in Baltimore, three presentations, reproduced here in an updated format, were delivered that, together, presented a different solution to this historical dilemma.

In the first paper, Noakes and St Clair Gibson ${ }^{9}$ present an historical overview of the evolution of the different models used to explain the physiological factors that are currently thought to limit human performance in exercise of either short duration and high intensity or lower intensity and longer duration. ${ }^{10-16}$ They pay special attention to the cardiovascular/anaerobic/catastrophic model because it is this model that has had the greatest influence on teaching and research in the exercise sciences ${ }^{17}$ since its first formulation in the early 1920s by AV Hill and his colleagues in London.

As astrophysicist Steven Hawking ${ }^{18}$ has written: "A theory is a good... model if it describes a wide class of observations and if it predicts the results of new observations. Beyond that it makes no sense to ask if it corresponds to reality, because we do not know what is reality independent of a theory. How can we know what is real, independent of a theory or model with which to interpret it?" Rather, the value of a model is that it makes predictions, the veracity of which can then be evaluated. If these predictions prove untrue, then the model must be relinquished: "Eventually the theory becomes a creaking and ugly edifice. Then someone suggests a new theory in which all the awkward observations are explained in an elegant and natural manner"

There are at least six hallmark requirements if the cardiovascular/anaerobic model is both plausible and defensible. Under all conditions of maximal exercise, there must be: (a) a "plateau" in oxygen consumption indicating $(b)$ the onset of skeletal muscle anaerobiosis; (c) a maximum (limiting) cardiac output; (d) complete (maximal) recruitment of all available motor units in the muscles that are exercising; (e) identical concentrations of inhibitory metabolites in the maximally recruited muscles at exhaustion regardless of the circumstances of the exercise; and $(f)$ an absolute state of fatigue from which complete recovery occurs only after a defined period of rest. As this model predicts that fatigue results from discrete system failure consequent to the attainment of a limiting cardiovascular function, the seventh prediction of this model is that there must be evidence of homoeostatic failure at fatigue, hence the development of a physiological "catastrophe". However, there is no conclusive evidence that fatigue during maximal exercise fulfils any, let alone all, of these predictions. Therefore the argument is advanced that this traditional model is a "creaking and ugly edifice ${ }^{\prime \prime 11}$ in need of urgent revision and reformulation.

The paper continues by evaluating the current models used to explain those forms of fatigue that develop during high intensity exercise of very short (less than 60 seconds), intermediate (minutes to hours), and very prolonged (two to eight hours) durations. It proposes that these current models also fail adequately to explain all the published findings. In particular, the model of fatigue during more prolonged exercise, which is believed to be due to muscle glycogen depletion causing "the rate of ATP production to fall below the rate of ATP use", ${ }^{6}$ cannot be correct. For the ultimate outcome must also be the development of rigor, which, as is the common observation, does not occur during prolonged exercise.

Hence Noakes and St Clair Gibson' ${ }^{9}$ conclude that the current models, which posit that fatigue is regulated exclusively by changes that occur in the exercising muscles, do not explain most forms of exercise fatigue. The most telling evidence for their conclusion is the finding that motor unit recruitment in the active muscles is never absolutely maximal $^{19-21}$ during voluntary exercise in humans. Yet the single absolute requirement of the traditional (peripheral) model of exercise regulation is that all the motor units in the active muscles must be recruited at exhaustion during all forms of exercise. However, there is neither any known nor proposed mechanism by which the accumulation or depletion of metabolites in the active muscles can directly prevent the fresh conscription of additional motor units that are quiescent at exhaustion because they have yet to be recruited by the central nervous system (CNS). For the conscription of additional motor units would allow the exercise to continue past a state of apparently absolute exhaustion but which is nonetheless associated with a less than maximum recruitment of available motor units.

More probable is the theory that such metabolites can more profitably act to regulate skeletal muscle function by influencing the number of motor units that are recruited in the exercising skeletal muscles at any time by the CNS. In essence, they argue that neither ATP nor lactic acid is the most important chemical regulating the function of exercising skeletal muscle, but rather the calcium released from the skeletal muscle sarcoplasmic reticulum in response to neural inputs from the central and peripheral nervous systems, which is also the essential chemical initiator of skeletal muscle contraction.

Accordingly, in the second paper, St Clair Gibson and Noakes $^{22}$ begin to establish the basis for a new model which they believe is better able to explain all the current observations. They argue that the peripheral model of fatigue is an example of a linear dynamic system in which system failure results from the progressive increase in the quantity of some, perhaps metabolic, variable until an absolute maximal concentration is reached. Once the system has failed catastrophically, causing physical exhaustion, a period of rest is required before exercise can again commence and during which the metabolites responsible for the fatigue are removed. However, they argue that, in humans with an intact CNS, no single metabolite has yet been identified that causes fatigue according to this linear model. More importantly, no example of absolute system failure, such as skeletal muscle rigor, has ever been reported; yet this is the logical prediction of the catastrophe model of Edwards. ${ }^{7}$

They next review the contrasting central neural or central governor model of fatigue, which is based on the evidence that there is always skeletal muscle motor unit "recruitment reserve" 1920 in all forms of fatigue, indicating that the CNS regulates and indeed limits the extent of skeletal muscle recruitment, specifically to ensure that homoeostasis is maintained and catastrophe averted.

This system would probably be controlled by either feed forward regulation from higher cortical structures or reflex inhibition of efferent neural command by type III and IV chemoreceptors reacting to changes in peripheral substrate or metabolite concentrations, or even perhaps from the input of skeletal, cardiac, or respiratory muscle mechanoreceptors. On the basis of a number of their $\mathrm{own}^{23}{ }^{24}$ and other ${ }^{25-27}$ recent findings, they next propose an updated and more comprehensive version of their original model, described by Noakes et al. ${ }^{28}$

According to their central governor model, they propose that during self paced exercise, which is the natural, as opposed to laboratory, form of human physical activity, the CNS continuously modifies the pace as part of a complex, non-linear dynamic system. In this model, the power output (work rate) during exercise is continuously adjusted on the basis of metabolic calculations performed at a subconscious CNS level. These calculations take into account prior knowledge acquired during previous exercise bouts, the planned end point of the current exercise bout, and the current metabolic rate, among many other potential variables. These 
subconscious calculations create a continuously adjusting power output, and hence pace, during exercise. This model provides the revolutionary insight that fatigue is not a physical event but rather a sensation that is the conscious manifestation of these subconscious CNS processes. ${ }^{20} 2930$

Thus the model predicts that the ultimate control of exercise performance resides in the brain's ability to vary the work rate and metabolic demand by altering the number of skeletal muscle motor units recruited during exercise. Thus the key question becomes: what physiological parameters determine the number of motor units recruited by the CNS in actively exercising skeletal muscles?

In the third paper, Lambert, St Clair Gibson, and Noakes ${ }^{31}$ examine peripheral regulation of metabolic activity, and how peripheral processes integrate with the regulatory centres in the CNS. They review the evidence showing that peripheral metabolites can provide information to central (brain) regulatory centres by way of afferent neural pathways and that they are therefore an integral part of the regulatory process. Alterations in efferent neural command generated as a result of peripheral afferent sensory input has a natural time delay, which leads to a continuously adjusting, oscillating power output, or pace, as part of a whole body, complex, non-linear, dynamic system.

Lambert, St Clair Gibson, and Noakes ${ }^{31}$ further argue that these alterations in power output may also be determined partly by regulatory systems that also exist in the periphery. These peripheral systems contribute to a hierarchical system of control with redundant properties, and this redundancy of command processes creates a more robust-that is, a complex-control system capable of maintaining homoeostasis more efficiently than would single, isolated control processes that lack a central integration or the presence of redundancies.

Finally, they show that the neural and metabolic responses to exercise may be "reset" by factors such as antecedent exposure to exercise. Even a single bout of exercise before a particular event can alter metabolic activity and the conscious sensation of fatigue during the subsequent exercise bout, indicating that the central governor processes are mutable and can be continuously reset on the basis of continuing and novel experiences.

The ideas presented here are revolutionary as opposed to evolutionary because they make three novel predictions.

- Firstly, as at rest, now also during all forms of exercise, all physiological functions are homoeostatically regulated by CNS control mechanisms to ensure that bodily harm does not result.

- Secondly, the conscious sensation of fatigue does not arise directly from the action of metabolites in the periphery, but rather from the regulatory centres in the subconscious parts of the brain, the function of which is to ensure homoeostasis during exercise. ${ }^{30}$ Therefore the distinct sensation of fatigue is not directly related to a physical end point, but is rather an interpretation of the effect of the current level of activity on future exercise capacity and any threats that immediate and future events pose to the maintenance of homoeostasis. ${ }^{30} 32$

- Thirdly, as the sensation of fatigue is an emotion rather than a physical state, pacing strategies and their control during self regulated exercise-the journey and not just the end point - are probably the most important phenomena in exercise physiology.

The authors therefore develop the argument that the "limitations" or "catastrophe" models of exercise physiology fail specifically because they do not explain what is most obvious. That far from being limited by the failure of one or
What is already known

A popular teaching is that exercise performance is limited by metabolic changes in the exercising muscles, so called peripheral fatigue. This model predicts that exercise terminates when there is a catastrophic failure of homoeostasis in the exercising muscles.

\section{What this study adds}

In a series of five articles introduced here, we present a revolutionary theory that explains the key findings better than the traditional catastrophe model of exercise physiology. The model proposes that exercise performance is regulated by the CNS specifically to ensure that catastrophic physiological failure does not occur during normal exercise in humans.

more physiological systems during exercise, the reality is that all physiological systems in the body, both at rest and during physical activity, are homoeostatically regulated in a continuous process, specifically to prevent catastrophic failure, including irreversible physical exhaustion, widespread cell damage, or even death, as the outcome of exercise.

\section{Authors' affiliations}

T D Noakes, A St Clair Gibson, E V Lambert, Research Unit for Exercise Science and Sports Medicine, University of Cape Town, Newlands, South Africa

\section{REFERENCES}

1 Gaitanos GC, Williams C, Boobis LH, et al. Human muscle metabolism during intermittent maximal exercise. J Appl Physiol 1993;75:712-19.

2 Newsholme EA, Start C. Regulation in metabolism. London: John Wiley and Sons, 1973:1-349.

3 Fitts RH. Cellular mechanisms of muscle fatigue. Physiol Rev 1994;74:49-94.

4 Rowland LP, Araki S, Carmel P. Contracture in McArdle's disease: stability of adenosine triphosphate during contracture in phosphorylase-deficient muscle. Arch Neurol 1965; 13:541-4.

5 Ruff RL. Why do patients with McArdle's disease have decreased exercise capacity? Neurology 1998;50:6-7.

6 Conlee RK. Muscle glycogen and exercise endurance: a twenty-year perspective. Exerc Sports Sci Rev 1987;15:1-28.

7 Edwards RHT. Biochemical bases for fatigue in exercise performance: catastrophe theory in muscular fatigue. In: Knuttgen HG, Vogel JA, Poortmans J, eds. Biochemistry of exercise. Champaign, IL: Human Kinetics, 1983:1-28.

8 Noakes TD. Physiological models to understand exercise fatigue and the adaptations that predict or enhance athletic performance. Scand J Med Sci Sports 2000; 10:123-45.

9 Noakes TD, St Clair Gibson A. Logical limitations in the "catastrophe" models of fatigue during exercise in humans. Br J Sports Med 2004

10 Noakes TD. Implications of exercise testing for prediction of athletic performance: a contemporary perspective. Med Sci Sports Exerc 1988;20:319-30

11 Noakes TD. Challenging beliefs: ex Africa semper aliquid novi. Med Sci Sports Exerc 1997;29:571-90.

12 Bassett DR, Howley ET. Maximal oxygen uptake: "classical" versus "contemporary" viewpoints. Med Sci Sports Exerc 1997;29:591-603.

13 Bassett DR, Howley ET. Limiting factors for maximum oxygen uptake and determinants of endurance performance. Med Sci Sports Exerc 2000;32:70-84.

14 Bergh U, Ekblom B, Astrand PO. Maximal oxygen uptake "classical" versus "contemporary" viewpoints. Med Sci Sports Exerc 2000;32:85-8.

15 Ekblom B. Editorial. Scand J Med Sci Sports 2000;10:119-22.

16 Noakes TD. Maximal oxygen uptake: "classical" versus "contemporary" viewpoints: a rebuttal. Med Sci Sports Exerc 1998;30:1381-98.

17 Mitchell JH, Saltin B. The oxygen transport system and maximal oxygen uptake. Chapter 6. In Exercise physiology. Oxford: Oxford University Press, 2003:255-91.

18 Hawking S. Black holes and baby universes and other essays. London: Bantam Press, 1993:1-173.

19 Gandevia SC. Spinal and supraspinal factors in human muscle fatigue. Physiol Rev 2001;81:1725-89.

20 St Clair Gibson A, Lambert MI, Noakes TD. Neural control of force output during maximal and submaximal exercise. Sports Med 2001;31:637-50. 
21 Ikai $\mathbf{M}$. Steinhaus $\mathrm{AH}$. Some factors modifying the expression of human strength. J Appl Physiol 1961;16:157-63.

22 St Clair Gibson A, Noakes TD. Evidence for complex system integration and dynamic neural regulation of skeletal muscle recruitment during exercise in humans. Br J Sports Med 2004

23 Kay D, Marino F, Cannon F, et al. Evidence for neuromuscular fatigue during high-intensity cycling in warm, humid conditions. Eur J Appl Physiol 2001;84:115-21.

24 St Clair Gibson A, Schabort EJ, Noakes TD. Reduced neuromuscular activity and force generation during prolonged cycling. Am J Physiol 2001;281:R187-96.

25 Ulmer H-V. Concept of an extracellular regulation of muscular metabolic rate during heavy exercise in humans by psychophysiological feedback. Experientia 1996;52:416-20.

26 Glass L. Synchronization and rhythmic processes in physiology. Nature $2001 ; 410: 277-84$
27 Lucia A, Hoyos J, Santalla A, et al. Tour de France versus Vuelta a Espana: which is harder? Med Sci Sports Exerc 2003;35:872-8.

28 Noakes TD, Peltonen JE, Rusko HK. Evidence that a central governor regulates exercise performance during acute hypoxia and hyperoxia. J Exp Biol 2001;204:3325-234.

29 St Clair Gibson A, Lambert EV, Lambert Ml, et al. Exercise and fatigue control mechanisms. Int J Sports Med 2001;2 (available from www.esportmed.com/ ismj//.

30 St Clair Gibson A, Baden DA, Lambert MI, et al. The conscious perception of the sensation of fatigue. Sports Med 2003;33:167-76.

31 Lambert EV, St Clair Gibson A, Noakes TD. Complex systems model of fatigue: integral control and defence of homeostasis during exercise in humans. Br J Sports Med 2004.

32 Tucker R, Rauch L, Harley $Y$, et al. Impaired exercise performance in the heat is associated with an anticipatory reduction in skeletal muscle recruitment. Pflugers Arch 2004 May 8 (Epub ahead of print).

\section{Call for papers}

10th European Forum on Quality Improvement in Health Care 13-15 April 2005, ExCel, Docklands, London

For further information on how to submit your paper please go to: http://www.quality.bmipg.com 